\title{
CYCLOTOMIC IDEALS IN GROUP RINGS
}

\author{
BY S. BACHMUTH AND H. Y. MOCHIZUKI
}

Communicated by Walter Feit, May 5, 1966

Let $G$ be a group of exponent $n$ and $Z(G)$ the integral group ring of $G$. We call the ideal of $Z(G)$, which is generated by all elements of the form $1+u+u^{2}+\cdots+u^{n-1}$ where $u$ runs through all elements of the group $G$ (including 1 ), the cyclotomic (or Burnside) ideal $\Im(n)$. The motivation for the study of these ideals is the close connection of $\Im(n)$ with the Burnside group $F / R^{\prime} F^{n}$ where $G=F / R$ is represented as a factor group of a free group, $R^{\prime}$ is the commutator subgroup of $R$, and $F^{n}$ is the group generated by $n$th powers of elements of $F$. Specifically, one considers $R F^{n} / R^{\prime} F^{n}$ as a module over $Z_{n}\left(F / R F^{n}\right)$ coupled with the observation that for $u$ in $R F^{n} / R^{\prime} F^{n}$ and $x$ in $F / R F^{n}$,

$$
u^{1+x+\cdots+x^{n-1}}=u\left(x u x^{-1}\right)\left(x^{2} u x^{-2}\right) \cdots\left(x^{n-1} u x^{-n+1}\right)=(u x)^{n} .
$$

We hope this announcement helps to initiate a study of the quotient rings $Z(G) / \Im(n)$. We restrict ourselves to the commutative case, where $G$ is a direct product of two cyclic groups of order $n$. We denote by $\Sigma$ the augmentation ideal of $Z(G)$; i.e., the ideal generated by $(1-g)$ for all $g$ in $G$. Throughout, the letter $F$ will always mean a free group of rank 2 .

\section{THEOREM 1.}

(i) $\Sigma^{p-1}=\Im(p),\left(\Sigma^{p-2} \subsetneq \Im(p)\right)$, for any prime $p$.

(i)i $\Sigma^{4} \subseteq \Im(4),\left(\Sigma^{3} \subsetneq \Im(4)\right)$.

(iii) $\Sigma^{12} \subseteq \Im(9),\left(\Sigma^{11} \subsetneq \Im(9)\right)$.

The application to the Burnside groups is the following:

Corollary 1.

(i) $F / F^{\prime \prime} F^{p}$ has nilpotency class $p-1$.

(ii) $F / F^{\prime \prime} F^{4}$ has nilpotency class 4 or 5 .

(iii) $F / F^{\prime \prime} F^{9}$ has nilpotency class 12 or 13 .

Part (i) of Corollary 1 is due to Meier-Wunderli [1], and Part (i) of Theorem 1 follows easily from his results. In general, our aim is to establish bounds on the nilpotency class of $F / F^{\prime \prime} F^{n}$ in the case where $n=p^{e}$ is a power of a prime, and in the case where $n$ is a composite, to determine where the lower central series becomes stationary. Since we can show the latter is determined once one knows the nilpotency class of $F / F^{\prime \prime} F_{i}^{e}$ for each prime power $p_{i}^{e}$ dividing $n$, we will restrict 
our attention to the case where $n=p^{e}$ for some prime $p$.

Theorem 1 provides, as far as our methods are concerned, the best possible information where the nilpotency class is determined within 1. (For the lower bounds we make use of the representation of $R / R^{\prime}$ as a $Z(F / R)$ module as given by Magnus [2].) To get this much information in the general situation seems difficult at the moment, but to get an error of $p$ appears to be relatively easy. For example, in the case $p^{3}=8$ and $p^{3}=27$, it is easy to establish $\Sigma^{13} \subseteq \Im(8)\left(\Sigma^{11} \Phi \Im(8)\right.$ ) and $\Sigma^{56} \subseteq \Im(27)\left(\Sigma^{53} \nsubseteq \Im(27)\right)$ which translates as follows: $F / F^{\prime \prime} F^{8}$ has nilpotency class at most 14 and at least 12 , while $F / F^{\prime \prime} F^{27}$ has nilpotency class at most 57 and at least 54 . However, the previous work makes one strongly suspect that $\Sigma^{54} \subseteq \Im(27)$ and $\Sigma^{12} \subseteq \Im(8)$. In the case of arbitrary $p^{2}$, we have shown the following:

THEOREM 2. $\Sigma^{2 p^{2}-p-1} \subseteq \Im\left(p^{2}\right)$ for arbitrary prime $p$.

Corollary 2. $F / F^{\prime \prime} F^{p^{2}}$ has nilpotency class at most $2 p^{2}-p$ and at least $2 p^{2}-2 p$.

For $p^{2}=4,9$, Theorem 1 gives sharper information, and in general, we believe the following to be the case.

CONJECTURE $1 . \Sigma^{e\left(p^{e}-p^{e-1}\right)} \subseteq \Im\left(p^{e}\right)$ for any prime $p$ and integer $e \geqq 1$.

$$
\left(\Sigma^{e(p e-p e-1)-1} \Phi \Im\left(p^{e}\right)\right) .
$$

A computation which yields results similar to Theorem 2 could be made for arbitrary $p^{e}$, but we have not done so yet. We can at this time state the following:

Theorem 3. For $x$ in $G,(1-x)^{e\left(p^{e-p^{0-1}}\right)}$ is in $\Im\left(p^{e}\right)$.

Corollary 3. $F / F^{\prime \prime} F^{p^{e}}$ has Engel lengthe $\left(p^{e}-p^{e-1}\right)-1$ or $e\left(p^{e}-p^{e-1}\right)$.

CoRollary 4. $F / F^{(k)} F^{p^{e}}$ has Engel length $\leqq(k-1) e\left(p^{e}-p^{e-1}\right)$.

Here $F^{(k)}$ denotes the $k$ th term of the derived series of $F$. The impetus for much of our work and for which Conjecture 1 would be an immediate corollary is

ConJeCture 2 . Let $\omega$ be a primitive $p^{e}$ root of unity and $Z_{p}{ }^{e}[\omega]$ $\cong Z_{p^{e}}[x] / \theta_{p^{e}}(x)$, where $\theta_{p^{e}}(x)$ is the irreducible polynomial which $\omega$ satisfies. Let $k$ be the kernel of a homomorphism of $Z(G)$ into $Z_{p}{ }^{e}[\omega]$. Let $K$ be the intersection of all such kernels. Then $K=\Im\left(p^{e}\right)$.

We have been able to prove Conjecture 2 only in simplest situations (e.g., $p=2,3,5$ ) and even in the simplest cases, where $Z_{p}{ }^{e}$ contains nilpotent elements (e.g., $p^{e}=4$ ), a great deal of complexity is introduced. 
Added in proof (September 1966). Conjecture 1 has been proved by Professor H. Heilbronn. Thus it follows that $F / F^{\prime \prime} F^{p^{e}}$ has nilpotency class $\left.e\left(p^{e}-p^{e-1}\right)\right)$ or $e\left(p^{e}-p^{e-1}\right)+1$.

\section{REFERENCES}

1. H. Meier-Wunderli, Metabelsche Gruppen, Comment. Math. Helv. 25 (1951), 1-10.

2. W. Magnus, On a theorem of Marshall Hall, Ann. of Math. (2) 40 (1939), 764-768.

University of California, Santa Barbara 